\title{
DESENVOLVIMENTO DE UM DISPOSITIVO PARA MEDIÇÃO DE ESFORÇOS EM HASTES DE SEMEADORAS DE PLANTIO DIRETO
}

\author{
AMAURI C. E. SANTO ${ }^{1}$, ÂNGELO V. DOS REIS ${ }^{2}$, ANTÔNIO L. T. MACHADO ${ }^{3}$, \\ VILSON J. BATISTA ${ }^{4}$, ANDRÉ OLDONI ${ }^{5}$
}

\begin{abstract}
RESUMO: Inúmeros problemas relativos à mecanização dizem respeito aos esforços demandados pelas máquinas e implementos agrícolas. A medição dos esforços em implementos de hastes é geralmente feita por instrumentos complexos e de difícil construção, como os anéis octogonais. Sendo assim, o objetivo deste artigo é apresentar o desenvolvimento de um dispositivo de medição de esforços horizontais e verticais em hastes sulcadoras de semeadoras-adubadoras de plantio direto, utilizando transdutores de força extensiométricos do tipo viga em balanço, comercialmente disponíveis. Para tanto, projetou-se um dispositivo tipo pórtico, composto de dois transdutores para medição de esforços horizontais e um transdutor para medição do esforço vertical. Os três transdutores foram montados de modo a restringir qualquer movimento da haste durante a interação com o solo. O protótipo construído foi submetido a ensaios estáticos em bancada de laboratório. Em um dos ensaios, aplicou-se carga horizontal na haste; no outro, aplicou-se carga vertical e no terceiro, a carga foi aplicada com inclinação de $15^{\circ}$ em relação à horizontal, para simular as condições de operação da haste no campo. O dispositivo mostrou-se robusto e, durante os testes de bancada, foi capaz de medir esforços horizontais de até $5.300 \mathrm{~N}$, e esforços verticais de até $2.000 \mathrm{~N}$, de forma linear e com grande coeficiente de correlação com as cargas aplicadas.
\end{abstract}

PALAVRAS-CHAVE: transdutor de força, ensaio de semeadoras, dinamômetro.

\section{DEVELOPMENT OF A DEVICE FOR MEASURING FORCES IN SHANKS OF NO-TILL PLANTERS}

\begin{abstract}
Many problems related to the mechanization involve the draft demanded by machinery and agricultural implements. The measurement of forces in shank implements is generally made by complex and difficult building instruments, as the octagonal ring. Therefore, the aim of this paper is to present the development of a device for measuring the horizontal and vertical forces in shanks of no-till planters by employing off the shelf shear beam force transducers. For this purpose a square shaped frame was designed with two transducers for measuring horizontal forces and another transducer to measure the vertical force. They were mounted with links that restrict the movement of the shank in any direction during its interaction with the soil. A prototype was built and submitted to static laboratory bench testings. One test was made with the application of horizontal load over the shank, another with the application of vertical load and the last one with the application of load with an inclination of $15^{\circ}$ to the horizontal to simulate the operating conditions of the shank on the field. The device proved to be robust and it was capable of measuring horizontal forces up to $5,300 \mathrm{~N}$ and vertical forces up to $2,000 \mathrm{~N}$, in a linear fashion and with a great correlation coefficient with the applied loads.
\end{abstract}

KEYWORDS: force transducer, planter testing, dynamometer.

\footnotetext{
${ }^{1}$ Prof. Associado, DEA/FEA/UFPel, Pelotas - RS, Fone: (0XX53) 3275.7126, amaurisanto@ hotmail.com

${ }^{2}$ Prof. Adjunto, DER/FAEM/UFPel, Pelotas - RS, Fone: (OXX53) 3275.7126, areis@ ufpel.edu.br;

${ }^{3}$ Prof. Associado, DER/FAEM/UFPel, Pelotas - RS, Fone (0XX53) 3275.7126, lilles@ ufpel.edu.br

${ }^{4}$ Prof. Associado, EE/UFRGS, Porto Alegre - RS, Fone: (0XX51) 3316.3567, vbatista@ mecanica.ufrgs.br

${ }^{5}$ Engenheiro Agrícola, andreoldoni@gmail.com

Recebido pelo Conselho Editorial em: 26-11-2008

Aprovado pelo Conselho Editorial em: 12-12-2009
} 


\section{INTRODUÇÃO}

O estudo dos esforços que atuam sobre as máquinas e implementos agrícolas vem a ser um dos parâmetros que maior contribuição promove para a solução dos problemas com mecanização no meio rural. A elaboração de projetos mais apropriados, a eliminação do desperdício e o uso adequado de máquinas e implementos dependem dessas informações (CASÃO JÚNIOR \& CHANG, 1993).

O esforço de tração e o tempo disponível normalmente ditam a potência necessária de um trator numa propriedade agrícola. Como o trator é o maior investimento de capital, o conhecimento do correto esforço de tração se faz necessário na tomada de decisão relativa às máquinas agrícolas. Além disso, a otimização da energia é um importante critério no projeto de máquina agrícola (MIELKE et al., 1994).

MACHADO (2001), na previsão do esforço de tração em ferramentas estreitas em solos do Rio Grande do Sul, partindo da instalação de extensômetros resistivos colados à haste de um escarificador, obteve os resultados referentes aos esforços de tração por meio de sistema coletor e processador de sinais com transferência para microcomputador.

CEPIK (2002), na análise do desempenho de uma haste sulcadora de semeadora-adubadora, como forma de coletar os dados a campo e determinar a força de tração na haste, utilizou de quatro extensômetros instalados no suporte da haste, de tal forma a medir a sua deformação quando em operação de campo. Os extensômetros foram ligados em Pontes de Wheaststone e conectados ao sistema de aquisição dos dados, permitindo a conversão dos valores de tensão em esforços atuantes na haste.

GODWIN (1975) desenvolveu um transdutor de força em forma de anel octogonal estendido para o estudo de ferramentas de preparo do solo. $\mathrm{O}$ autor partiu da premissa de que o sistema de forças atuantes no preparo do solo, onde a falha do solo é simétrica, consiste em duas componentes de forças perpendiculares mutuamente, sendo uma força horizontal, outra vertical e um momento no plano dessas duas forças. SOUZA \& MAGALHÃES (1989) apresentaram um transdutor de força baseado na metodologia de cálculo de GODWIN (1975) para a análise de esforços horizontais e verticais em hastes de escarificadores.

Observando as condições de contorno estabelecidas para o dimensionamento feito por SOUZA \& MAGALHÃES (1989), BORDIGNON (2005) verificou que o equipamento de medição projetado por esses autores, mesmo tendo sido para hastes escarificadoras, poderia atender à análise de esforços em semeadoras-adubadoras. Considerou o dimensionamento feito e utilizou os valores por eles obtidos para a construção do elemento elástico do sensor. E, a partir das características estáticas nominais de trabalho, ou seja, força horizontal máxima de $19,6 \mathrm{kN}$ e força vertical máxima de 7,84 kN, e com um momento máximo de $13,05 \mathrm{kNm}$, desenvolveu e analisou a implementação de transdutores de força, do tipo anel octogonal estendido, para auxiliar na avaliação de esforços em máquinas agrícolas, sobretudo em semeadoras-adubadoras.

A análise dos trabalhos dos pesquisadores que utilizaram anéis octogonais estendidos mostra que, embora os resultados obtidos sejam plenamente satisfatórios, o projeto e a construção desses equipamentos são complexos, exigindo conhecimentos de resistência dos materiais, extensometria e eletrônica, além de capacidade construtiva. Outra possibilidade para a medição de esforços em hastes é a utilização de transdutores de força extensométricos comercialmente disponíveis, arranjados de forma a medir, independentemente, as forças horizontais e verticais observadas. Acredita-se que essa iniciativa não tenha surgido anteriormente pelo fato de os transdutores de força serem equipamentos caros até algum tempo atrás. Na atualidade, dada a maior oferta, esses sensores tornaram-se mais acessíveis e a um custo menor.

$\mathrm{O}$ inconveniente dessa abordagem é a impossibilidade de medir o momento associado às forças vertical e horizontal e, com isso, não poder determinar o ponto de aplicação da força resultante na ponteira de trabalho. No entanto, o desconhecimento desse parâmetro não inviabiliza o 
uso de um instrumento assim construído para a determinação do esforço de tração demandado pelo conjunto haste-ponteira, permitindo que seja empregado em inúmeras situações práticas da pesquisa.

Sendo assim, o objetivo deste artigo é apresentar o desenvolvimento de um dispositivo de medição de esforços horizontais e verticais em hastes sulcadoras de semeadoras-adubadoras de plantio direto, utilizando transdutores de força extensométricos do tipo viga em balanço, comercialmente disponíveis.

\section{MATERIAL E MÉTODOS}

Para que transdutores de força do tipo viga em balanço sejam utilizados para medir todos os esforços na direção de deslocamento de uma haste, é necessário que não haja transmissão de carregamentos entre eles no momento de medir a força resultante da interação com o solo. Para tanto, o dispositivo de medição dos esforços (Figura 1) foi projetado com três transdutores de força fabricados em aço inoxidável, sendo dois com capacidade nominal de $50 \mathrm{kN}$, montados paralelamente, e um de $15 \mathrm{kN}$ disposto ortogonalmente em relação aos outros dois, no mesmo plano médio. Como princípio e como forma de possibilitar a medição das cargas atuantes, os mesmos foram vinculados à haste por meio de um conjunto de peças especialmente construído. Esta disposição possibilita, por meio dos dois transdutores dispostos verticalmente, a determinação da força horizontal e, através do transdutor de força montado na posição horizontal, o esforço vertical, ambos atuantes na ponteira sulcadora.

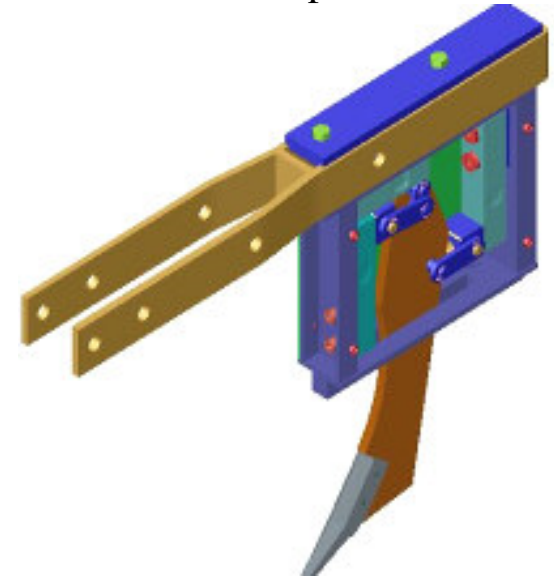

(a)

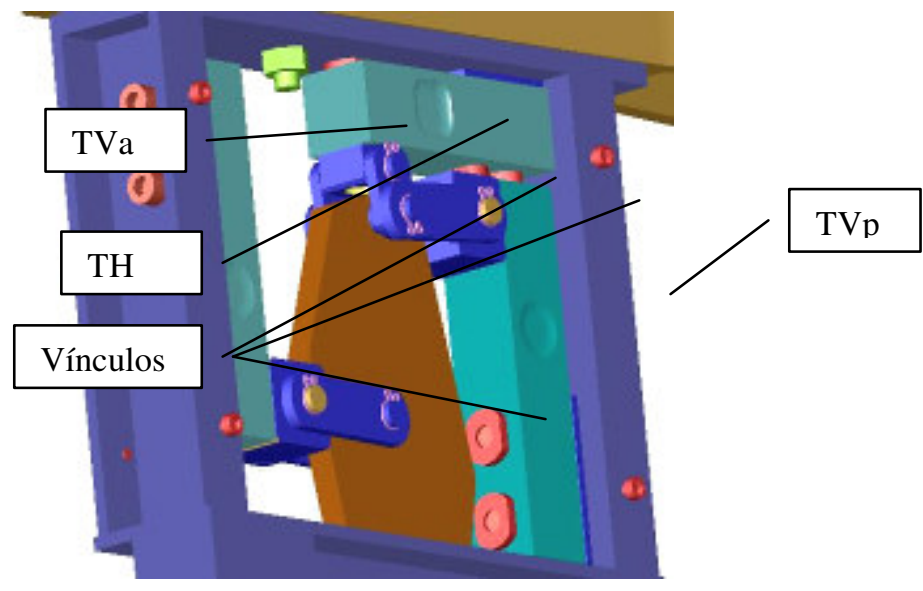

(b)

FIGURA 1. Vista do conjunto e detalhe do dispositivo projetado em CAD, em que: (a) vista geral do conjunto; (b) detalhes de montagem dos transdutores e de seus vínculos com a haste, em que: TVa - transdutor vertical anterior; TVp - transdutor vertical posterior; $\mathrm{TH}$ - transdutor horizontal. General view and detail of the device designed on CAD sofware, where: (a) general view, (b) assembly details of transducers and their links with the shank, where: TVa - front vertical transducer; TVp - rear vertical transducer; TH - horizontal transducer.

Na Figura 2a, é apresentado o modelamento dos vínculos estruturais existentes entre os três transdutores de força do dispositivo e a haste sulcadora. Todos os três vínculos restringem o movimento apenas na direção de medição de esforços de cada transdutor. Assim, os transdutores, dispostos verticalmente (TVa e TVp), medem apenas forças horizontais, enquanto o transdutor instalado horizontalmente (TH), acima da haste, mede apenas os esforços verticais. Na Figura $2 b$, tem-se a representação das forças envolvidas quando a ponteira da haste abre o sulco no solo.

A partir da representação esquemática (Figura 2b), podem-se estabelecer as equações que determinam as forças horizontal e vertical atuantes na ponteira, em função do ângulo de inclinação 
da haste sulcadora $(\alpha)$, considerando-se as condições de equilíbrio nas direções horizontal e vertical, conforme desenvolvimento apresentado nas eqs. (1) a (13).

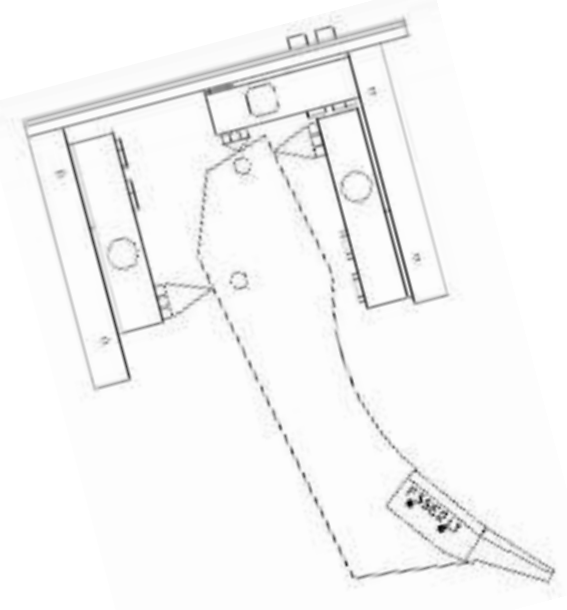

a

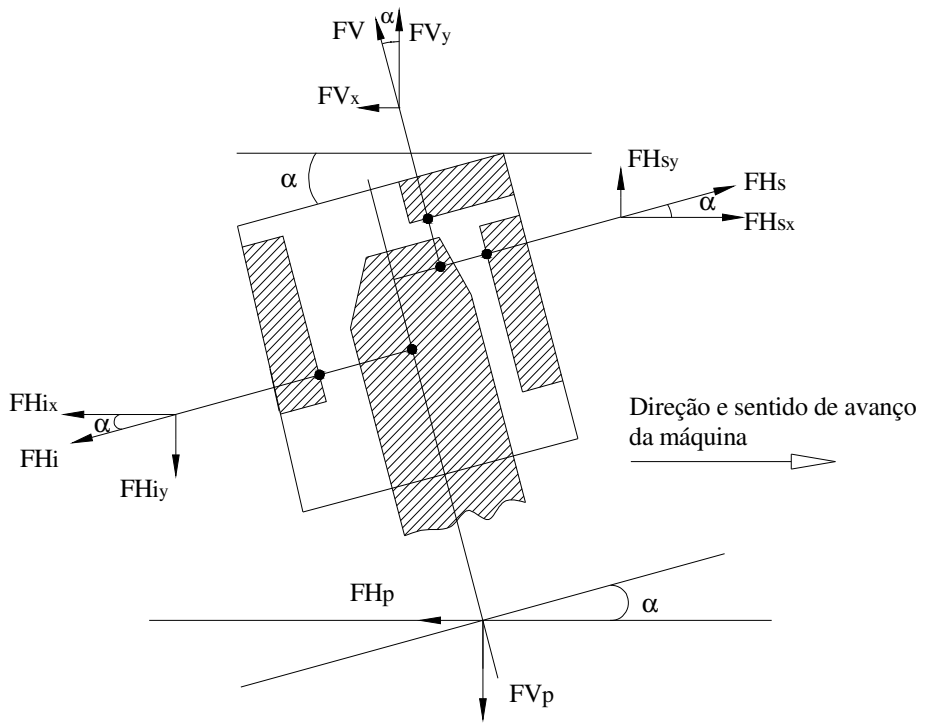

b

FIGURA 2. Representação esquemática das forças atuantes, em que: (a) vínculos estruturais entre a haste e os transdutores, e (b) forças envolvidas. Schematic representation of the acting forces, where: (a) structural links between the shank and the transducers and (b) involved forces.

Considera-se que:

$$
\mathrm{FH}_{\mathrm{P}}+\mathrm{FHi}_{\mathrm{x}}+\mathrm{FV}_{\mathrm{x}}=\mathrm{FHs}_{\mathrm{x}}
$$

logo,

$$
\text { FHP }=\text { FHsx }- \text { Fhix }- \text { FVx }
$$

como,

$$
\begin{aligned}
& \mathrm{FHs}_{\mathrm{x}}=\mathrm{FHs} \cos \alpha \\
& \mathrm{Fhi}_{\mathrm{x}}=\text { Fhi } \cos \alpha \\
& \mathrm{FV}_{\mathrm{x}}=\mathrm{FV} \operatorname{sen} \alpha \\
& \mathrm{RH}=\text { FHs }- \text { Fhi }
\end{aligned}
$$

então,

$$
\mathrm{FH}_{\mathrm{P}}=(\mathrm{FHs}-\mathrm{Fhi}) \cos \alpha-\mathrm{FV} \operatorname{sen} \alpha
$$

logo,

$$
\mathrm{FH}_{\mathrm{P}}=\mathrm{RH} \cos \alpha-\mathrm{FV} \operatorname{sen} \alpha
$$

De maneira análoga:

$$
\mathrm{FV}_{\mathrm{P}}+\mathrm{Fhi}_{\mathrm{y}}=\mathrm{FV}_{\mathrm{y}}+\mathrm{FHs}_{\mathrm{y}}
$$

ou,

$$
\text { FVP }=\text { FHsy }- \text { Fhiy }+ \text { FVy }
$$

então,

$\mathrm{FV}_{\mathrm{P}}=\mathrm{FHs}$ sen $\alpha-$ Fhi sen $\alpha+\mathrm{FV} \cos \alpha$

logo, 


$$
\mathrm{FV}_{\mathrm{P}}=(\mathrm{FHs}-\mathrm{Fhi}) \operatorname{sen} \alpha+\mathrm{FV} \cos \alpha
$$

Assim sendo,

$$
\mathrm{FV}_{\mathrm{P}}=\mathrm{RH} \operatorname{sen} \alpha+\mathrm{FV} \cos \alpha
$$

em que,

$\mathrm{FH}_{\mathrm{P}}$ e $\mathrm{FV}_{\mathrm{P}}$ - forças horizontal e vertical atuantes sobre a ponteira sulcadora, $\mathrm{N}$;

$\mathrm{RH}$ - resultante das leituras de TVa e TVp, N;

FV - leitura de TH, N;

FHs e Fhi - leituras de TVa e TVp, N;

$\mathrm{FV}_{\mathrm{x}}$ e $\mathrm{FV}_{\mathrm{y}}$ - respectivamente, componentes horizontal e vertical da leitura de $\mathrm{TH}, \mathrm{N}$;

$\mathrm{FHs}_{\mathrm{x}}$ e $\mathrm{Fhi}_{\mathrm{x}}$ - componentes horizontais das leituras de TVa e TVp, N, e

$\mathrm{FHs}_{\mathrm{y}}$ e $\mathrm{Fhi}_{\mathrm{y}}$ - componentes verticais das respostas de TVa e TVp, N.

Assim, com o uso das eqs.(8) e (13), é possível transformar as leituras nos transdutores do dispositivo de medição nas forças horizontal e vertical que atuam na ponteira sulcadora.

Durante o teste de campo do dispositivo, o ângulo de inclinação da haste sulcadora ( $\alpha$ ) foi medido indiretamente com auxílio de esquadro de marceneiro e réguas, com a haste e a instrumentação já montadas na semeadora e na posição de trabalho.

Como forma de adaptação e proteção física das células de carga, foram montadas duas tampas retangulares sobre as laterais do pórtico estrutural. Essas, construídas em chapa de Aço SAE 1020, com dimensões tais que formaram uma caixa de adaptação do aparato de medição, e de posicionamento da haste sulcadora na condição original da semeadora (Figura 3).

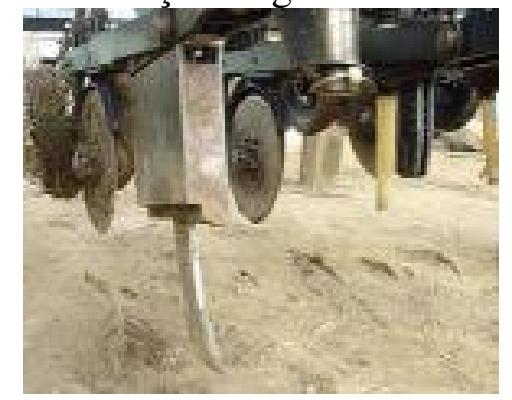

FIGURA 3. Dispositivo de medição com haste e ponteira montado na linha da semeadora. The measuring device with shank and tip mounted on the planter row.

Para a verificação da interferência cruzada causada pelas forças horizontal e vertical medidas pelos respectivos transdutores de força, principalmente pela ação dos vínculos existentes entre a haste e os transdutores, o dispositivo foi submetido à ação de cargas individuais e ortogonais (direção horizontal e direção vertical). Já para verificar a existência de interferência cruzada entre os sensores na posição de operação, executou-se uma simulação em bancada para reproduzir a condição de trabalho durante a realização dos testes de campo. Para a realização dos ensaios estáticos, construíram-se diversos dispositivos, peças e suportes especiais para a fixação do dispositivo de medição à estrutura metálica da bancada de testes. Vide Figuras 4a, b e c.

Nas três condições de ensaio estático, ou seja, horizontal, vertical e inclinada, utilizou-se um transdutor de força do tipo "S", com capacidade de $20 \mathrm{kN}$. Esse sensor foi previamente aferido e, após, utilizado como padrão de referência secundário para medir os valores de carga, que foram aplicados por meio de um esticador do tipo rosca. 


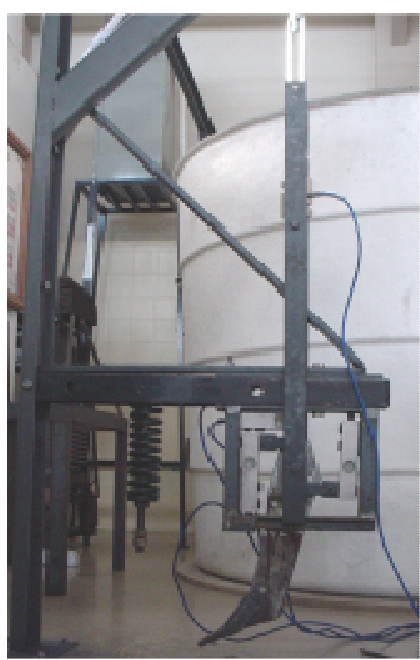

a

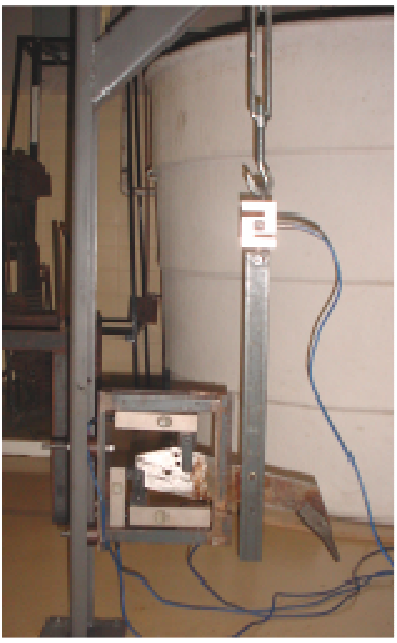

b

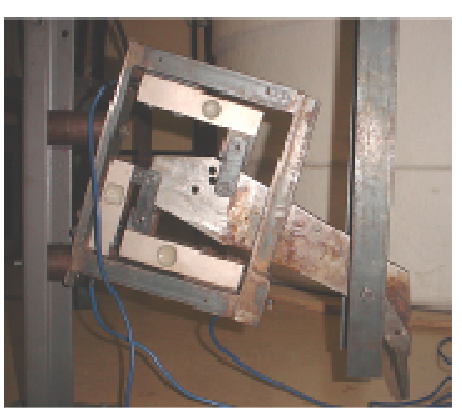

c

FIGURA 4. Detalhamento da montagem utilizada para o ensaio do dispositivo: (a) esforços verticais; (b) esforços horizontais; (c) esforços horizontais e verticais (dispositivo posicionado na condição de trabalho - inclinação de $15^{\circ}$ ). Details of the assembly used to test the apparatus: (a) vertical forces; (b) horizontal forces; (c) horizontal and vertical forces (device positioned as on fieldwork - inclination of $\left.15^{\circ}\right)$.

O processo, aplicação de cargas crescentes e decrescentes, determinado como padrão, foi realizado com três repetições em cada etapa do ensaio, utilizando-se de uma taxa de aquisição de $30 \mathrm{~Hz}$ para a amostragem do sinal de cada um dos transdutores. Nos ensaios de campo, utilizou-se uma taxa de aquisição de $300 \mathrm{~Hz}$. Para os ensaios de aferição do dispositivo para as condições de aplicação de esforços puramente horizontais e verticais, aplicaram-se cargas de até 5.300 N. Já no ensaio com o mecanismo inclinado, a carga máxima aplicada foi de $4.500 \mathrm{~N}$. Esses valores excedem aqueles que são esperados em hastes de sulcadoras de semeadoras de plantio direto.

Os dados registrados durante cada uma das três repetições, das três condições de ensaio, foram submetidos à análise de regressão para que fosse possível inferir sobre a linearidade entre as cargas aplicadas e os valores medidos nos transdutores e o grau de correlação entre eles.

Após o ensaio estático de laboratório, o dispositivo, juntamente com a haste, foi montado numa semeadora para a realização de teste de campo em condições normais de operação. Trabalhou-se à velocidade média de $1,39 \mathrm{~m} \mathrm{~s}^{-1}\left(5 \mathrm{~km} \mathrm{~h}^{-1}\right)$ e uma semeadora-adubadora marca Vence-Tudo, modelo SA9400, com 9 linhas espaçadas de 0,45 m. O teste de campo teve a finalidade de observar a montagem do dispositivo na semeadora e de verificar como se dariam a leitura e o registro dos esforços induzidos pela abertura do sulco através da haste. A profundidade de trabalho foi regulada para $0,12 \mathrm{~m}$.

O trabalho de campo foi realizado numa área de campo nativo, localizada no município de Pelotas-RS, nas seguintes coordenadas geográficas: latitude $31^{\circ} 52^{\prime} 00^{\prime}$ ' sul e longitude 52 $21^{\prime} 24^{\prime}$ ' oeste. O solo é classificado como Planossolo Hidromórfico (STRECK et al., 1999), com a seguinte distribuição granulométrica: $0,611 \mathrm{~kg} \mathrm{~kg}^{-1}$ de areia, $0,257 \mathrm{~kg} \mathrm{~kg}^{-1}$ de silte e $0,132 \mathrm{~kg} \mathrm{~kg}^{-1}$ de argila.

Para a aquisição de sinais, foi utilizada uma unidade da marca Lynx, modelo ADS20000IP, composto por conversor A/D e controlador AC2122 de 16 bits, condicionador de sinais AI2164 contendo 32 canais de entrada configuráveis por software. Para o registro dos dados, foi utilizado um microcomputador portátil da marca Toshiba, modelo Satélite.

Os transdutores de carga empregados são calibrados de fábrica e apresentam as características mostradas na Tabela 1. 
TABELA 1. Características de desempenho dos transdutores empregados no dispositivo. Performance features of the transducers used in the device.

\begin{tabular}{|c|c|}
\hline Parâmetro & Valor \\
\hline$\overline{\text { Saída nominal (mV/V) }}$ & 3,00 \\
\hline Zero inicial (\% S.N.) ${ }^{*}$ & $+/-1,00$ \\
\hline Não linearidade (\% S.N.) & 0,03 \\
\hline Histerese (\% C.N.) ${ }^{* *}$ & 0,02 \\
\hline Não repetibilidade (\% S.N.) & 0,02 \\
\hline Creep à capacidade nominal (\% de carga em $\left.20^{\prime}\right)$ & 0,03 \\
\hline
\end{tabular}

${ }^{*}$ Saída nominal; ${ }^{* *}$ Capacidade nominal

\section{RESULTADOS E DISCUSSÃO}

Nos ensaios estáticos, têm-se os resultados com aplicação de carga vertical, carga horizontal e de ambas, simultaneamente, apresentados nas Figuras 5; 6 e 7, respectivamente. Em cada uma, é possível observar o comportamento do sinal de resposta do transdutor que mede força na mesma direção de aplicação da carga, como também dos transdutores que lhe são ortogonais.

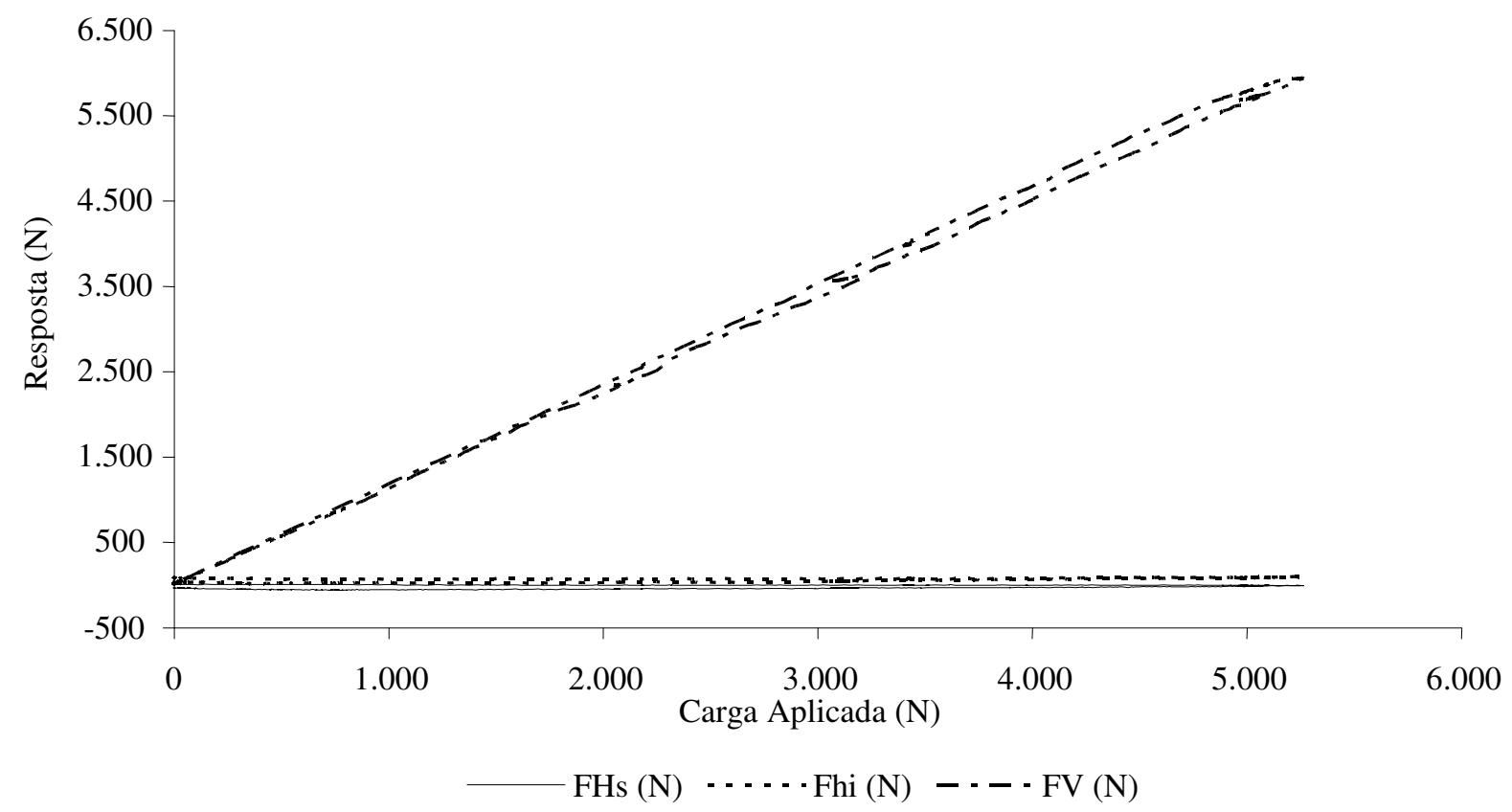

FIGURA 5. Exemplo de ensaio com aplicação de carga vertical. Sample testing with the application of vertical load.

O ensaio com aplicação de carregamentos verticais puros, ou seja, na mesma direção de leitura do transdutor vertical, mostrou (Figura 5) que a forma de fixação dos transdutores, assim como a sua disposição, conseguiu isolar a transmissão de esforços na direção horizontal. As leituras, em ambos os transdutores horizontais, permaneceram em torno de zero até a capacidade nominal. 


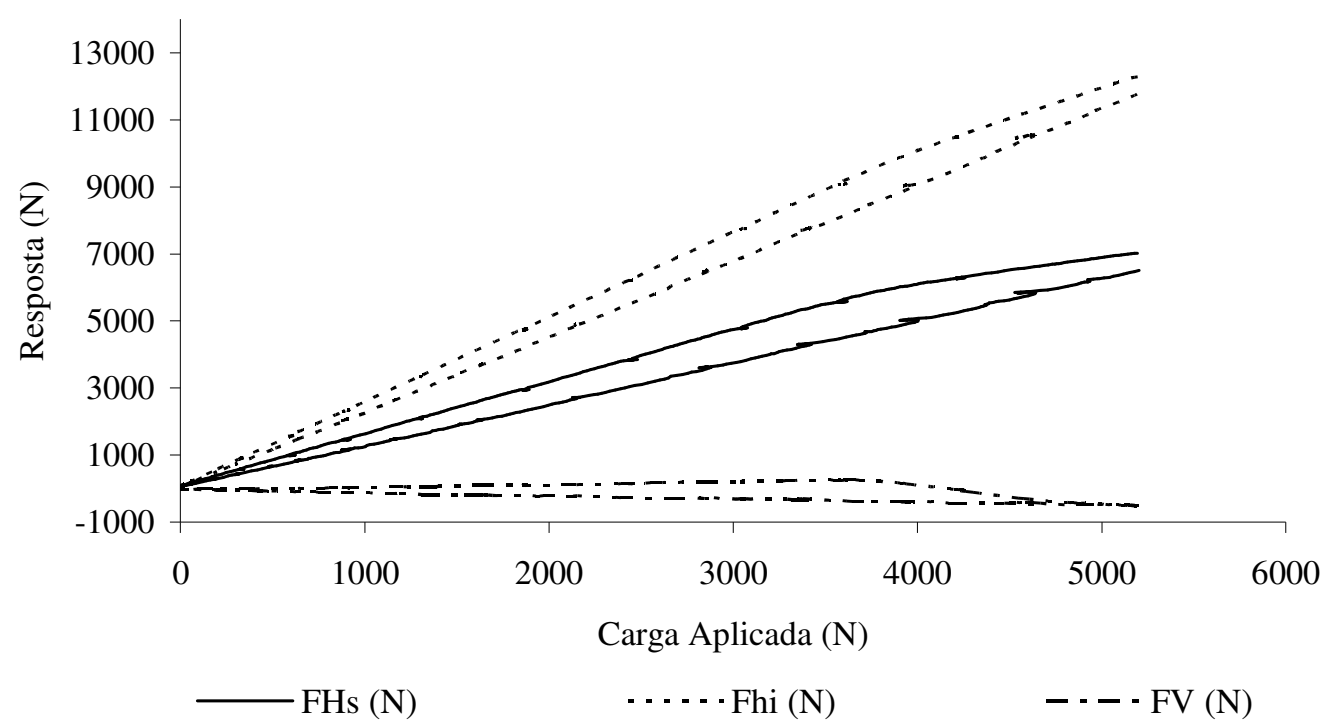

FIGURA 6. Exemplo de ensaio com aplicação de carga horizontal. Sample testing with the application of horizontal load.

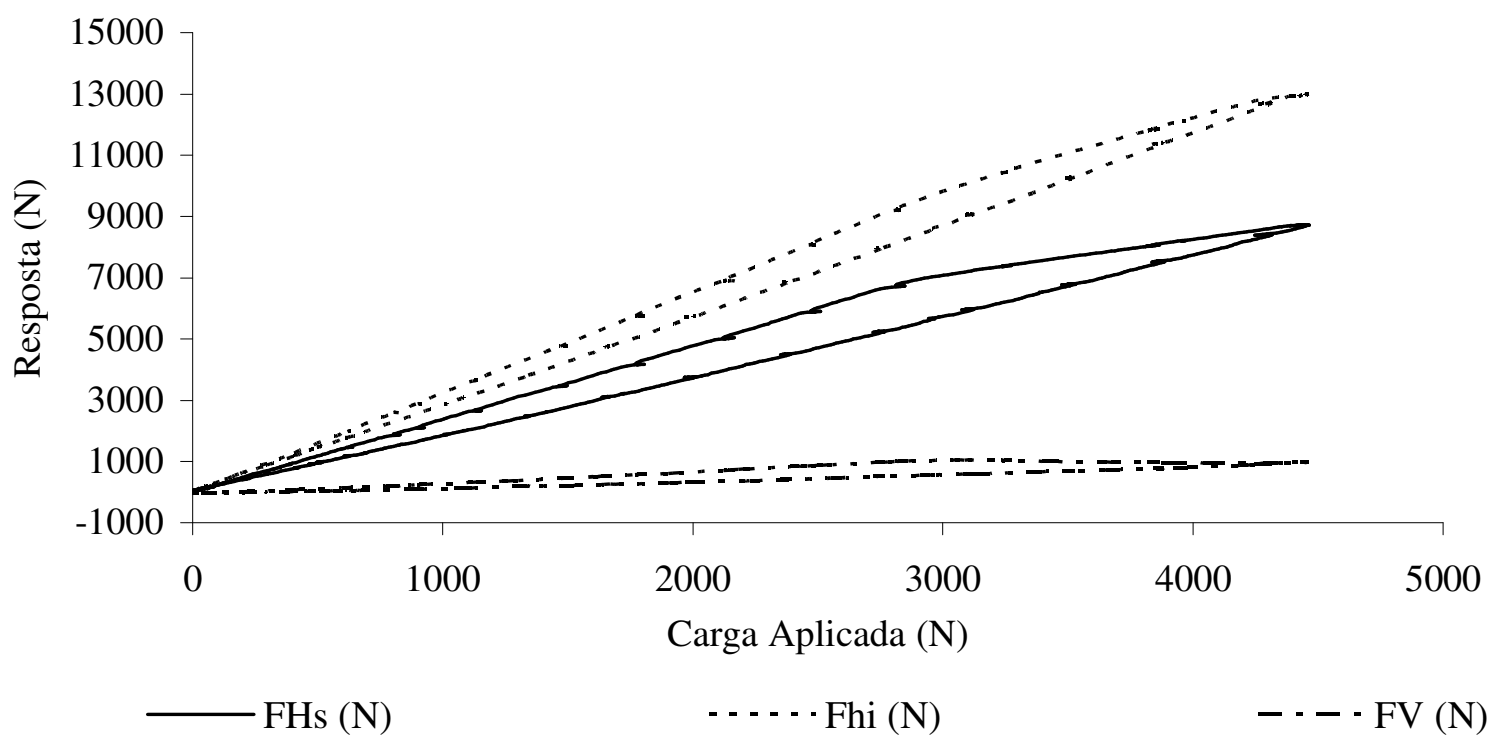

FIGURA 7. Exemplo de ensaio com aplicação de carga na situação de campo ( $15^{\circ}$ de inclinação). Sample testing with the application of load as in the field $\left(15^{\circ}\right.$ inclination $)$.

A análise de regressão feita entre a carga vertical aplicada e a força vertical medida (FV) mostrou boa correlação e ajuste linear (Tabela 2). As três repetições feitas em todas as condições de ensaio de laboratório permitiram que fossem calculadas, para a aplicação de cargas vertical e horizontal separadamente, algumas características de desempenho do dispositivo, como erros de histerese, repetibilidade, não linearidade e erro total combinado, os quais também são apresentados na Tabela 2.

O ensaio com aplicação de carga horizontal, ou seja, na direção de leitura dos transdutores horizontais, mostrou (Figura 6), como era de se esperar, que os valores lidos no transdutor posterior (Fhi) são maiores que os lidos no transdutor anterior (Fhs). No entanto, a força horizontal resultante [(ver eq.(6)] apresentou boa correlação com as cargas aplicadas, como se pode observar na Tabela 2. 
TABELA 2. Análises de regressão entre as cargas aplicadas e os valores medidos nos três transdutores, nas três situações ensaiadas, e características de desempenho do dispositivo. Regression analyses between the applied loads and the measured values in the three transducers in the three tested cases and the performance features of the device.

\begin{tabular}{|c|c|c|c|c|c|c|}
\hline Carga Aplicada (x) & $\begin{array}{l}\text { Equação de } \\
\text { Ajuste }\end{array}$ & $\mathrm{R}^{2}$ & $\begin{array}{l}\text { Repetibilidade } \\
\text { (\% S.N.) }\end{array}$ & $\begin{array}{c}\text { Histerese } \\
(\% \text { S.N.) }\end{array}$ & $\begin{array}{c}\text { Não } \\
\text { Linearidade } \\
(\% \text { S.N. })\end{array}$ & $\begin{array}{c}\text { Erro Total } \\
\text { Combinado } \\
(\% \text { S.N. })\end{array}$ \\
\hline Vertical & $\mathrm{FV}=1,148 \mathrm{x}$ & 0,9995 & 1,59 & 4,32 & 11,67 & 12,52 \\
\hline Horizontal & $\mathrm{RH}=1,005 \mathrm{x}$ & 0,9995 & 1,82 & 1,59 & 1,26 & 1,61 \\
\hline $\begin{array}{l}\text { Inclinação } 15^{\circ} \\
\text { Componente vertical }\end{array}$ & $\mathrm{FV}=0,900 \mathrm{x}$ & 0,8636 & - & - & - & - \\
\hline $\begin{array}{l}\text { Componente } \\
\text { horizontal }\end{array}$ & $\mathrm{RH}=1,010 \mathrm{x}$ & 0,9960 & - & - & - & - \\
\hline
\end{tabular}

O erro total combinado, observado na força horizontal resultante, correspondente ao esforço de tração na haste de uma semeadora, quando da aplicação de carga horizontal, não ultrapassou $1,61 \%$ do sinal nominal de saída.

O esforço medido no transdutor vertical permaneceu próximo de zero até a carga horizontal aplicada atingir $4.000 \mathrm{~N}$. A partir desse ponto, pode-se observar redução no valor da força vertical. $\mathrm{O}$ desvio máximo verificado foi de $-469 \mathrm{~N}$ para carga horizontal aplicada de $5.000 \mathrm{~N}$, correspondendo a 9,30\% do sinal de saída. Esta dificuldade foi evidência pelos valores altos de não linearidade e erro total combinado apresentados na Tabela 2. Não foi possível determinar as causas da interferência da carga horizontal aplicada, no transdutor vertical; entretanto, especula-se que o atrito interno dos vínculos ou a deformação da estrutura do dispositivo, com cargas mais elevadas, podem ter causado o sinal medido no canal vertical.

No ensaio feito aplicando-se carga com inclinação de $15^{\circ}$, simulando a condição de operação da ponteira no campo, verificou-se, mais uma vez, bom ajuste linear da força horizontal resultante com a componente horizontal da carga aplicada (Tabela 2). A equação de ajuste da força vertical medida e da componente vertical da carga aplicada denota a interferência da montagem mecânica no resultado lido, conforme discutido anteriormente.

Com os resultados dos ensaios estáticos, é possível verificar que o dispositivo é capaz de medir com acuidade esforços horizontais de até $5.300 \mathrm{~N}$. Esse valor, segundo MACHADO et al. (2007), é aproximadamente o dobro do resultado médio encontrado pela pesquisa de esforço de tração em semeadoras no Brasil (esforço de tração de $2.680 \mathrm{~N}$ por linha) e aquém da recomendação de tração dos fabricantes de semeadoras (esforço de tração de $7.300 \mathrm{~N}$ por linha). Considerando que o dispositivo mede apenas o esforço necessário para o deslocamento de uma haste, e não do restante da linha, considera-se que o mesmo é adequado às pesquisas de campo nessa área.

No que se refere aos esforços verticais induzidos pelo deslocamento da ferramenta no solo, verificou-se que os valores lidos têm sua validade limitada a esforços horizontais de até $4.000 \mathrm{~N}$. A partir desse valor, há interferência entre os transdutores. Utilizando-se dos dados da aferição, foi possível determinar que, para uma carga vertical de $2.000 \mathrm{~N}$, o erro total combinado é de 4,59\% S.N. Esses erros são crescentes, e para uma carga de $2.500 \mathrm{~N}$ já atingem 7,07\% S.N.

\section{Teste de campo}

O teste de campo do dispositivo, juntamente com o sistema de aquisição de dados, transcorreu sem problemas. A forma construtiva da caixa medidora permitiu, de maneira rápida e simples, não só a montagem do dispositivo à estrutura da semeadora-adubadora, como também a operação de troca das ponteiras durante os testes, sem a necessidade de retirada do equipamento da máquina. 
Além disso, possibilitou a manutenção da real posição de trabalho do conjunto haste-ponteira sulcadora em sua forma original.

No momento do teste, o solo apresentava índice de cone de $4.824 \mathrm{kPa}$ na faixa de profundidade de zero a $0,12 \mathrm{~m}$ e teor de água de $0,13 \mathrm{~kg} \mathrm{~kg}^{-1}$.

Na Figura 8, é apresentada a tela de saída do programa de aquisição de dados. É possível observar, de forma qualitativa, o comportamento da máquina durante o período de teste. Entre 0 e $5 \mathrm{~s}$ (abscissas), tem-se o momento em que a máquina foi baixada e a ponteira tocou o solo. De 5 a aproximadamente $11 \mathrm{~s}$, permaneceu parada. A partir desse momento, tem-se o início do deslocamento, percebendo-se a região de aceleração do conjunto e, após, o regime de operação na condição plena com a velocidade constante (aproximadamente de 11 a $31 \mathrm{~s}$ ).
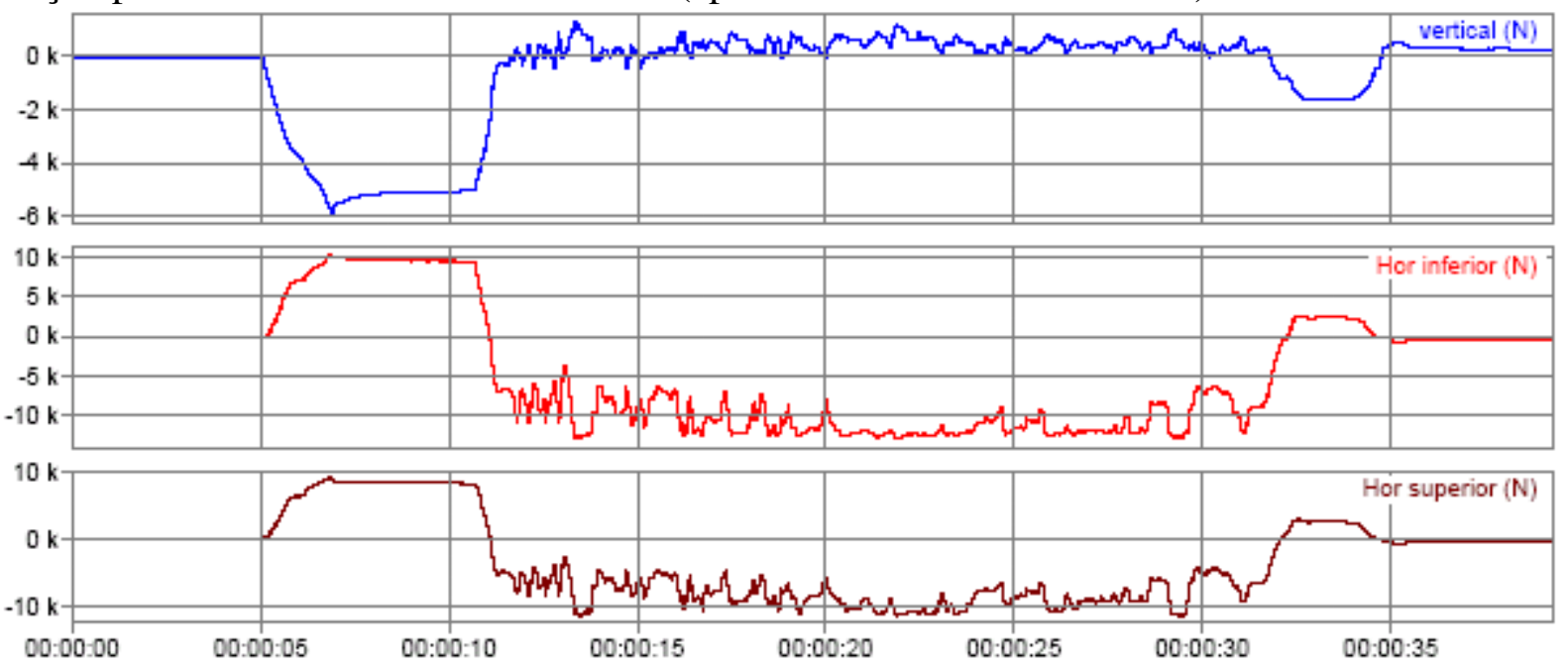

FIGURA 8. Tela de saída do programa de aquisição de dados com o registro das forças atuantes nos transdutores do dispositivo durante o teste de campo. Screen output of the acquisition software with the record of the acting forces on transducers of the device during the field test.

Durante o teste de campo, verificou-se a facilidade de operação do dispositivo e do sistema eletrônico, permitindo rapidez e eficiência nas operações de campo e na aquisição de dados. Foi observada, ainda, a capacidade de combinar o sistema implementado para atender às demandas de diversas pesquisas, podendo ser utilizado em diferentes tipos de semeadoras-adubadoras, bastando para isso alterar o sistema de fixação à linha da máquina.

Por fim, o dispositivo mostrou-se com baixo custo de confecção, pois o seu valor total montado, incluindo os três transdutores de força, material de construção mecânica, elementos de fixação e eletrodos de solda, foi inferior a R \$ 3.100,00 (US\$ 1.600,00). Não foram incluídos os custos de usinagem (hora-homem e hora-máquina), pois a construção deu-se nas oficinas das instituições envolvidas no projeto. Além disso, apresenta como vantagem a exatidão oferecida pelas células de carga utilizadas. Outro aspecto a ser considerado é a praticidade de reposição das células de carga, visto que, por serem comerciais, são de fácil aquisição, não exigindo demanda de tempo em projeto, construção e calibração.

\section{CONCLUSÕES}

O dispositivo projetado com três transdutores de força do tipo viga em balanço foi capaz de medir esforços horizontais de até $5.300 \mathrm{~N}$ e esforços verticais de até $2.000 \mathrm{~N}$, de forma linear e com grande coeficiente de correlação com as cargas aplicadas.

O sistema de vínculos entre os transdutores, e entre esses e a haste, foi capaz de evitar a transmissão cruzada indesejada de sinais entre os transdutores verticais (medição de força horizontal), e o transdutor horizontal para cargas horizontais de até $4.000 \mathrm{~N}$. 
Não houve interferência na força horizontal medida, a qual permaneceu em torno de zero, quando cargas verticais de até $5.300 \mathrm{~N}$ (capacidade nominal do dispositivo) foram aplicadas.

O dispositivo pode ser montado com facilidade na linha da semeadora, permitindo que a substituição de ponteiras fosse feita normalmente, não havendo nenhum tipo de prejuízo ao trabalho da máquina.

\section{REFERÊNCIAS}

BORDIGNON, J. Projeto e construção de sensores de força para medição de esforços em semeadoras-adubadoras. 2005. 141 f. Dissertação (Mestrado em Engenharia Mecânica) Faculdade de Engenharia Mecânica, Universidade Federal do Rio Grande do Sul, Porto Alegre, 2005.

CASÃO JÚNIOR, R.; CHANG, C.S. Estudo dos esforços tridimensionais em hastes de paraplow em caixa de solo. In: CONGRESSO BRASILEIRO DE ENGENHARIA AGRÍCOLA, 22., 1993, Ilhéus. Anais... Ilhéus: Sociedade Brasileira de Engenharia Agrícola, 1993. p.1.878-1.894.

CEPIK, C.T. Análise do desempenho de uma haste sulcadora-adubadora, em diferentes teores de água no solo, velocidades e profundidades de trabalho. 2002. $63 \mathrm{f}$. Dissertação (Mestrado em Ciência do Solo) - Faculdade de Agronomia, Universidade Federal do Rio Grande do Sul, Porto Alegre, 2002.

GODWIN, R.G. An extend octogonal ring transducer for use in tillage studies. Journal of Agricultural Engineering Research, St. Joseph, n.20, p.347-352, 1975.

MACHADO, A.L.T. Previsão do esforço de tração para ferramentas estreitas em solos do Rio Grande do Sul. 2001. 172 f. Tese (Doutorado em Ciência do Solo) - Faculdade de Agronomia, Universidade Federal do Rio Grande do Sul, Porto Alegre, 2001.

MACHADO, R.L.T.; FERREIRA, M.F.P.; MACHADO, A.L.T.; REIS, A.V.; BUTZKE, H.P. Força de tração de semedoras para plantio direto: informações de campo x informações de catálogo. Tecno-Lógica, Santa Cruz do Sul, v.11, n.1, p.10-11, 2007.

MIELKE, L.N.; GRISSO, R.D.; BASHFORD, L.L.; PARKHURST, A.M. Bi-level subsoiler performance using tanden shanks. Applied Engineering in Agriculture, St. Joseph, v.10, n.3, p.345349, 1994.

SOUZA, W.R.; MAGALHÃES, P.S.G. Projeto e construção de um transdutor para teste de implementos agrícolas. In: CONGRESSO BRASILEIRO DE ENGENHARIA AGRÍCOLA, 18., 1989, Recife. Anais... Jaboticabal: Sociedade Brasileira de Engenharia Agrícola, 1989. v.1, p.295306.

STRECK, E.V.; KAMPF, N.; KLAMT, E. Atualização da classificação taxonômica das unidades de mapeamento do levantamento de reconhecimento dos solos do estado do Rio Grande do Sul. Porto Alegre: EMATER, 1999. (Informativo, 5) 